\title{
Spatial postprocessing of ensemble forecasts for temperature using nonhomogeneous Gaussian regression
}

\author{
Kira Feldmann, Michael Scheuerer ${ }^{\dagger}$ and Thordis L. Thorarinsdottir ${ }^{\ddagger}$
}

\begin{abstract}
Statistical postprocessing techniques are commonly used to improve the skill of ensembles of numerical weather forecasts. This paper considers spatial extensions of the well-established nonhomogeneous Gaussian regression (NGR) postprocessing technique for surface temperature and a recent modification thereof in which the local climatology is included in the regression model for a locally adaptive postprocessing. In a comparative study employing $21 \mathrm{~h}$ forecasts from the COSMO-DE ensemble predictive system over Germany, two approaches for modeling spatial forecast error correlations are considered: A parametric Gaussian random field model and the ensemble copula coupling approach which utilizes the spatial rank correlation structure of the raw ensemble. Additionally, the NGR methods are compared to both univariate and spatial versions of the ensemble Bayesian model averaging (BMA) postprocessing technique.
\end{abstract}

\section{Introduction}

The first ensemble prediction systems were developed in the early 1990s to account for the various sources of uncertainty in the numerical weather prediction (NWP) model outputs (Lewis, 2005). Such systems have now become the state-of-the-art in meteorological forecasting (Leutbecher and Palmer, 2008). Additionally, the ensemble forecasts are commonly postprocessed using statistical techniques to improve the calibration and correct for potential biases, and a diverse range of postprocessing techniques has been proposed (e.g. Gneiting et al., 2005; Raftery et al., 2005; Wilks and Hamill, 2007; Bröcker and Smith, 2008). While these methods have been shown to greatly improve the predictive performance, many are only applicable to univariate weather quantities and neglect forecast error dependencies over time or between different observational sites. However, correct multivariate dependence structure is often important in applications, especially when considering composite quantities such as minima, maxima or an aggregated total. These quantities are crucial e.g. for highway maintenance operations or flood management, where subsequent risk calculations based on the forecast require a calibrated probabilistic forecast for both the original weather variable and the composite quantity.

In this paper, we focus on spatial extensions of the nonhomogeneous Gaussian regression (NGR) or ensemble model output statistics method for surface temperature, originally proposed by Gneiting et al. (2005). NGR is a parsimonious postprocessing technique which, for temperature, returns a Gaussian predictive distribution where the mean value is an affine function of the ensemble member forecasts while the variance is an affine function of the ensemble variance. The parameters of the model are estimated based on recent forecast errors jointly over a region or separately at each observation location (Gneiting et al., 2005; Thorarinsdottir and Gneiting, 2010). Other applications of this approach include Hagedorn et al. (2008) and

\footnotetext{
${ }^{*}$ Ruprecht-Karls-Universität Heidelberg, Germany

${ }^{\dagger}$ National Oceanic and Atmospheric Administration, Boulder, Colorado, U.S.A.

${ }^{\ddagger}$ Norwegian Computing Center, Oslo, Norway
} 
Kann et al. (2009). Recently, Scheuerer and König (2014) proposed a modification of the NGR methods of Gneiting et al. (2005) in which the postprocessing at individual locations varies in space by parameterizing the predictive mean and variance in terms of the local forecast anomalies rather than the forecasts themselves.

To obtain a multivariate predictive distribution based on a deterministic temperature forecast, Gel et al. (2004) propose the geostatistical output perturbation (GOP) method to generate spatially consistent forecasts fields in which the forecast error field is described through a Gaussian random field model. Berrocal et al. (2007) combine GOP with the univariate postprocessing method ensemble Bayesian model averaging (BMA) of Raftery et al. (2005). Ensemble BMA for temperature dresses each bias corrected ensemble member with a Gaussian kernel and returns a predictive distribution given by a weighted average of the individual kernels. By merging ensemble BMA and GOP, calibrated probabilistic forecasts of entire weather fields are produced. We propose a similar conceptualization, combining the NGR methods of Gneiting et al. (2005) and Scheuerer and König (2014) with a Gaussian random field error model in an approach we refer to as spatial NGR.

As an alternative multivariate method, we consider the non-parametric ensemble copula coupling (ECC) approach of Schefzik et al. (2013). ECC returns a postprocessed ensemble of the same size as the original raw ensemble. The prediction values at each location are samples from the univariate postprocessed predictive distribution at that location. Multivariate forecast fields are subsequently generated using the rank correlation structure of the raw ensemble. ECC thus assumes that the ensemble prediction system correctly describes the spatial dependence structure of the weather quantity. The method applies equally to any multivariate setting and comes at a virtually no additional computational cost once the univariate postprocessed predictive distributions are available.

Figure 1 illustrates temperature field forecasts obtained from the raw ensemble, the standard univariate NGR method, NGR combined with ECC, and spatial NGR. The raw ensemble is depicted in the first row. The NWP model output has a physically consistent spatial structure, but as we shall see later, it is strongly underdispersive and does not adequately represent the true forecast uncertainty. The samples in rows 2-4 all share the same NGR marginal predictive distributions which have larger uncertainty bounds than the raw ensemble. In the second row, the realizations have been sampled independently for each gridpoint i.e. no spatial dependence structure is present. This results in unrealistic temperature fields and, when considering compound quantities, forecasts that are statistically inappropriate. The combination of NGR and ECC in the third row gives forecast fields with similar spatial structures as the raw ensemble even though there is larger spread both within each field and between the realized fields. As a consequence of spatial correlations being modeled through a discrete copula, the resulting temperature fields feature some sharp transitions at locations where the ranks of the raw ensemble change. The bottom row depicts temperature field simulations obtained with spatial NGR. Here, the spatial dependence between forecast errors at different locations is modeled by a statistical correlation model and the physical consistency is implicitly learned from the data.

In a comparative study, we apply the various extensions of NGR as well as ensemble BMA to $21 \mathrm{~h}$ forecasts of surface temperature over Germany issued by the German Weather Service through their COSME-DE ensemble prediction system. The remainder of the paper is organized as follows. The forecast and observation data are described in the next Section 2. The univariate NGR postprocessing methods are introduced in Section 3 while the multivariate methods are described in Section 4. In the following Section 5 we report the results of the case study and a discussion is provided in Section 6. Finally, an overview over the forecast evaluation methods applied in Section 5 is given in the Appendix.

\section{Forecast and observation data}

The COSMO-DE forecast dataset consists of a 20-member ensemble. Forecasts are made for lead times from 0 to $21 \mathrm{~h}$ on a $2.8 \mathrm{~km}$ grid covering Germany with a new model run being started every $3 \mathrm{~h}$. The ensemble is based on a convection-permitting configuration of the NWP model COSMO (Steppeler et al., 2003, Baldauf 

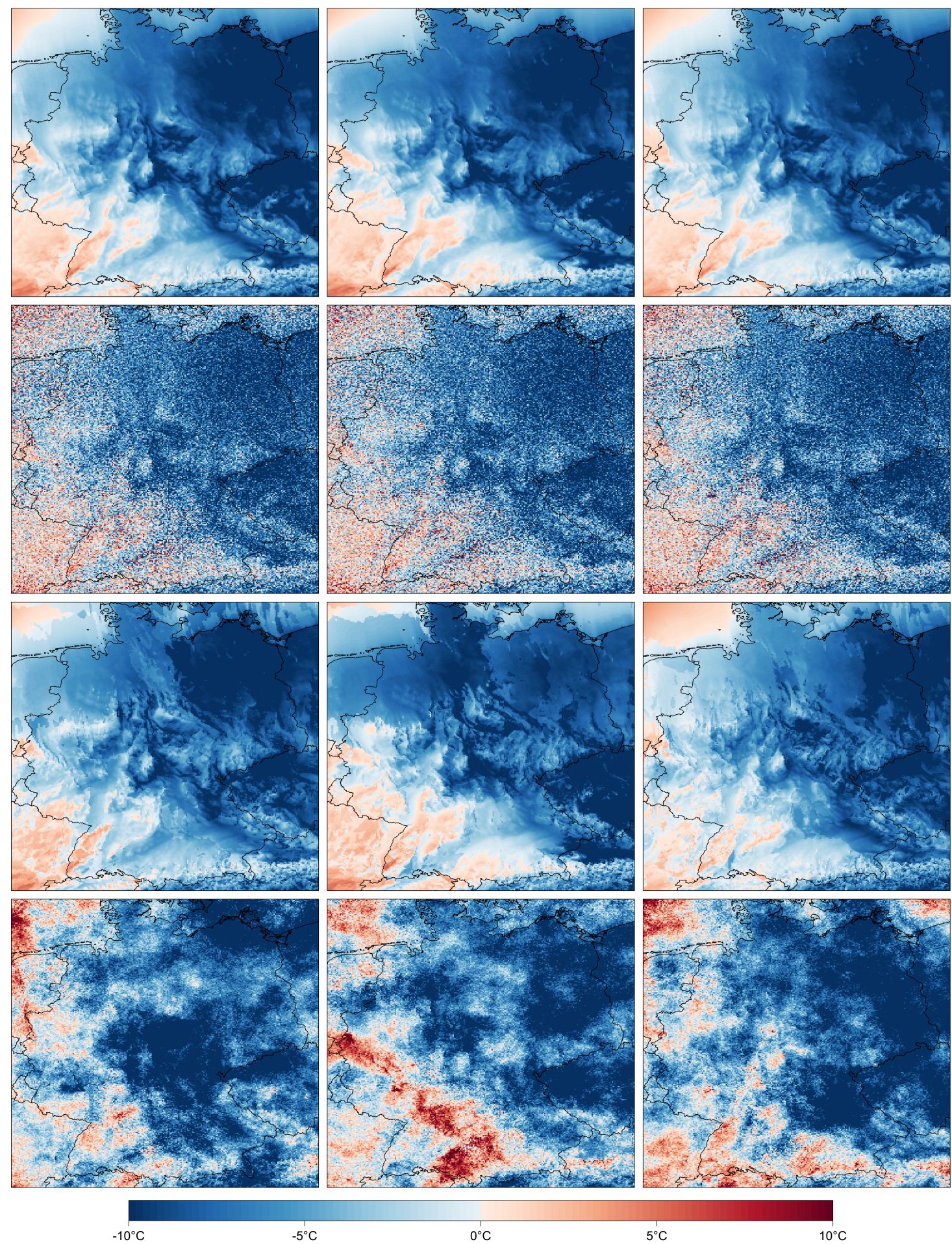

$5^{\circ} \mathrm{C}$

$10^{\circ} \mathrm{C}$

Figure 1: $21 \mathrm{~h}$ temperature forecasts over Germany for January 5, 2011 at 21:00UTC. Three members from the COSMO-DE ensemble prediction system are shown in the top row, the second row demonstrates the postprocessed nonhomogeneous Gaussian regression (NGR) forecast, the third row shows NGR combined with ensemble copula coupling while the bottom row displays examples of the multivariate spatial NGR forecast. 


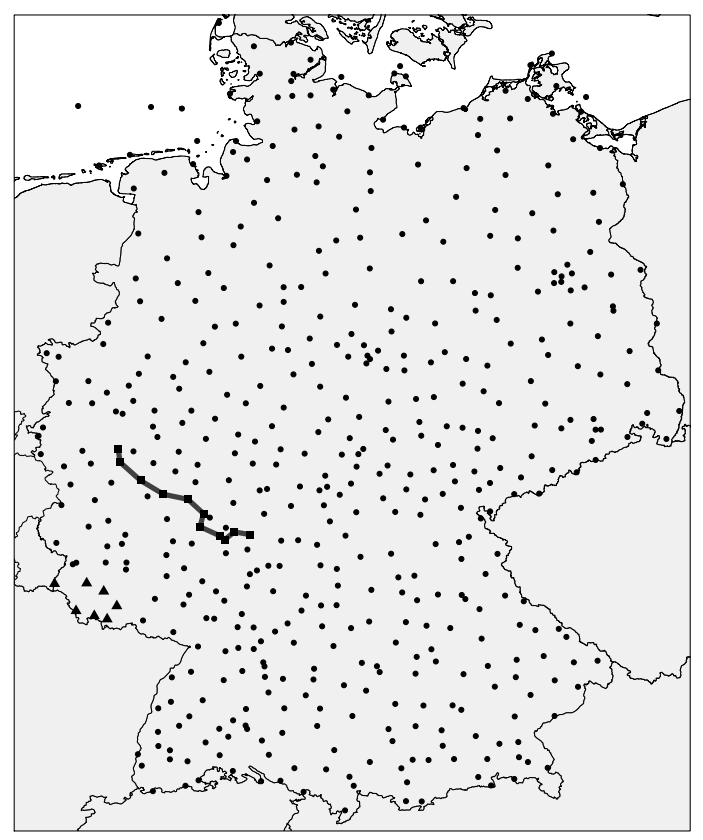

Figure 2: Map of Germany showing the location of a total of 514 SYNOP observation stations. The gray line illustrates a section of the highway A3 with the surrounding observation stations indicated by solid squares. Seven observation stations in the state of Saarland are represented by solid triangles. Other stations are indicated by solid circles.

et al., 2011). It has a $5 \times 4$ factorial design with 5 different perturbations in the model physics and 4 different initial and boundary conditions provided by global forecasting models (Gebhardt et al., 2011; Peralta and Buchhold, 2011). The pre-operational phase of the COSMO-DE ensemble prediction system started on 9 December 2010 and the operational phase was launched on 22 May 2012.

We employ $21 \mathrm{~h}$ forecasts from the pre-operational phase initialized at 00:00 UTC; our entire dataset consists of forecasts from 10 December 2010 until 30 November 2011. As we use a rolling training period of 25 days to fit the parameters of the statistical postprocessing methods, the evaluation period runs from 5 January 2011 to 30 November 2011. If at least one ensemble member forecast is missing at all observation locations on a specific day, we omit this day from the dataset. This way, 10 days are eliminated with 346 days remaining. The temperature observations we employ stem from 514 SYNOP stations over Germany. Their locations are shown in Figure 2. The forecasts are interpolated from the forecast grid to the station locations using bilinear interpolation. In total, we evaluate forecasts for 117,879 verifying observations over 346 days. The COSMO model uses a rotated spherical coordinate system in order to project the geographical coordinates to the plane with distortions as small as possible (Doms and Schättler, 2002, Sec. 3.3), with $421 \times 461$ equidistant gridpoints in longitudinal and latitudinal direction. We adopt this coordinate system to calculate horizontal distances within the framework of our spatial correlation models.

\section{Univariate postprocessing}

\subsection{NGR for temperature}

The NGR method of Gneiting et al. (2005) generalizes the common model output statistics (MOS) postprocessing technique, see e.g. Wilks (2011). The distribution of the future state $y_{s}$ of the temperature at location $s$ is modeled as a Gaussian distribution with parameters depending on the $M$ ensemble forecasts 
$f_{1 s}, \ldots, f_{M s}$,

$$
y_{s} \mid f_{1 s}, \ldots, f_{M s} \sim \mathcal{N}\left(a+b_{1} f_{1 s}+\ldots+b_{M} f_{M s}, c+d S_{s}^{2}\right),
$$

where $S_{s}^{2}$ is the ensemble variance, $a, b_{1}, \ldots, b_{M} \in \mathbb{R}$ are regression coefficients and $c, d \in \mathbb{R}_{+}$are nonnegative coefficients. NGR is thus a linear model with the ensemble forecasts as predictors and a nonhomogeneous error term which is modeled as an affine function of the ensemble variance $S_{s}^{2}$. This modeling set-up counteracts a possible over- or underdispersion of the ensemble while exploiting a positive spread-error correlation. The normal predictive distribution presents a reasonable model for variables like temperature or surface pressure. While the Gaussian assumption is not appropriate for all weather variables, the basic idea of NGR can also be used with other types of predictive distributions (Thorarinsdottir and Gneiting, 2010, Thorarinsdottir and Johnson, 2012; : Lerch and Thorarinsdottir, 2013; : Scheuerer, 2014).

In the formulation in (1), the regression coefficients $b_{1}, \ldots, b_{M}$ can take any value in $\mathbb{R}$. However, as negative values are difficult to interpret, Gneiting et al. (2005) suggest an alternative formulation restricting the coefficients $b_{1}, \ldots, b_{M}$ to be non-negative by iteratively removing those ensemble members $f_{m}$ from the linear model for which the coefficients $b_{m}$ are negative. We follow Thorarinsdottir and Gneiting (2010) and obtain non-negative coefficients by setting $b_{1}=\beta_{1}^{2}, \ldots, b_{M}=\beta_{M}^{2}$ with $\beta_{1}, \ldots, \beta_{M} \in \mathbb{R}$. The (normalized) coefficients can then be interpreted as weights and reflect the relative performance of the ensemble members during the training period. In the following, we refer to this approach as $\mathrm{NGR}_{+}$.

\subsection{Locally adaptive NGR}

The NGR postprocessing in (1) makes the same adjustments of ensemble mean and variance at all locations. However, it has been argued that systematic model biases may vary in space due to e.g. incomplete resolution of the orography or different land use characteristics. Similarly, the prediction uncertainty may differ between locations in a way that is not represented by the ensemble spread (Kleiber et al., 2011; Scheuerer and Büermann, 2014; Scheuerer and König, 2014).

As an alternative to the NGR model in (11), we consider the locally adaptive NGR method of Scheuerer and König (2014). Here, the local adaptation is obtained by incorporating information about the short-term local climatology in the covariates of the predictive distribution, thereby using forecast anomalies rather then the original forecasts as covariates. Specifically, let $\bar{y}_{s}^{\mathcal{T}}$ denote the average observed local temperature at location $s$ over all days $t$ in the training period $\mathcal{T}$ and, correspondingly, denote by $\bar{f}_{m s}^{\mathcal{T}}$ the average temperature forecast by the $m$-th ensemble member. The predictive distribution then equals

$$
y_{s} \mid f_{1 s}, \ldots, f_{M s}, \mathcal{D}_{s}^{\mathcal{T}} \sim \mathcal{N}\left(\bar{y}_{s}^{\mathcal{T}}+b_{1}\left(f_{1 s t}-\bar{f}_{1 s}^{\mathcal{T}}\right)+\ldots+b_{M}\left(f_{M s}-\bar{f}_{M s}^{\mathcal{T}}\right), c \xi_{s}^{2}+d S_{s}^{2}\right),
$$

where $\mathcal{D}_{s}^{\mathcal{T}}$ denotes the forecast and observation data at location $s$ during the training period and $\xi_{s}^{2}$ is a predictor variable for the location specific uncertainty. The location specific uncertainty is defined in a second step, when the regression estimates $\hat{b}_{1}, \ldots, \hat{b}_{M}$ are available. It is given by the mean squared residuals of the regression fit,

$$
\xi_{s}^{2}=\frac{1}{|\mathcal{T}|} \sum_{t \in \mathcal{T}}\left(y_{s t}-\bar{y}_{s}^{\mathcal{T}}-\sum_{m=1}^{M} \hat{b}_{m}\left(f_{m s t}-\bar{f}_{m s}^{\mathcal{T}}\right)\right)^{2},
$$

where $|\mathcal{T}|$ denotes the number of days in the training period. At locations without an observation station, predictive means and variances are obtained by spatial interpolation as described in Scheuerer and König (2014). We refer to this inclusion of the local climatology as $\mathrm{NGR}_{\mathrm{c}}$.

\subsection{Parameter estimation in the univariate setting}

We focus on the $\mathrm{NGR}_{+}$formulation of the NGR method in (1) and the $\mathrm{NGR}_{\mathrm{c}}$ extension in (2). The parameter estimation for both methods proceeds in a similar manner. It is assumed that the forecast error statistics 
change only slowly over time and a rolling training window $\mathcal{T}$ of the $|\mathcal{T}|$ most recent dates with forecasts and observations available is used to estimate the model parameter. The model fitting is carried out with all available data from the set of observation sites $\mathcal{S}$ within the training window $\mathcal{T}$. We denote this set by $\mathcal{S}$ in the equations below even though observations might not always be available at all observation sites. Note that with $\mathrm{NGR}_{+}$we can easily generate postprocessed forecasts outside the set $\mathcal{S}$ since the parameters of the postprocessing techniques are not site-specific. When $\mathrm{NGR}_{\mathrm{c}}$ is used, this can be achieved through an additional spatial interpolation step.

We follow Gneiting et al. (2005) and estimate the NGR + parameters by minimizing the continuous ranked probability score (CRPS) (e.g. Gneiting and Raftery, 2007) over the training set. That is, we chose them as a solution to

$$
\min _{a, b_{1}, \ldots, b_{M}, c, d} \frac{1}{|\mathcal{T}||\mathcal{S}|} \sum_{t \in \mathcal{T}} \sum_{s \in \mathcal{S}} \operatorname{crps}\left(\Phi_{s t}, y_{s t}\right),
$$

where $\Phi_{s t}$ is the Gaussian distribution function in (1) on training day $t$ at site $s, y_{s t}$ is the corresponding verifying observation and

$$
\operatorname{crps}\left(\Phi_{s t}, y_{s t}\right)=\int_{-\infty}^{\infty}\left(\Phi_{s t}(x)-\mathbf{1}_{\left[y_{s t}, \infty\right)}(x)\right)^{2} d x
$$

with $\mathbf{1}_{\left[y_{s t}, \infty\right)}(x)$ equal to 1 if $x \in\left[y_{s t}, \infty\right)$ and 0 otherwise. For Gaussian distributions, the integral in (4) can be expressed in a closed form which minimizes the computational costs (Gneiting et al., 2005). Software for the estimation and prediction is available through the ensembleMOS package in $\mathrm{R}$ (R Core Team, 2013) which is available at www.r-project.org.

The parameters of the $\mathrm{NGR}_{\mathrm{c}}$ method, on the other hand, are estimated in two steps. In a first step, the regression parameters $b_{1}, \ldots, b_{M}$ in (2) are estimated by weighted least squares using a penalized version of the loss function to prevent overfitting, see Scheuerer and König (2014) for details. The parameters of the variance function, $c$ and $d$, are subsequently estimated via CRPS minimization as in (3) above.

\section{Multivariate methods}

\subsection{Ensemble copula coupling}

The ensemble copula coupling (ECC) method of Schefzik et al.(2013) employs the rank order structure of the raw ensemble to obtain a postprocessed ensemble of forecasts fields with the same multivariate correlation structure as the raw ensemble while retaining the univariate NGR marginals. It is thus a semi-parametric copula approach with continuous marginals and the non-parametric empirical copula. For each $s \in \mathcal{S}$, we draw a sample of size $M$ from the predictive distribution $\hat{\Phi}_{s}$ given by (1) or (2) of the form

$$
\hat{f}_{1 s}=\hat{\Phi}_{s}^{-1}\left(\frac{1}{M+1}\right), \ldots, \hat{f}_{M s}=\hat{\Phi}_{s}^{-1}\left(\frac{M}{M+1}\right) .
$$

That is, it holds that $\hat{f}_{1 s} \leq \cdots \leq \hat{f}_{M s}$. Let $\rho_{s}$ denote a permutation of the integers $\{1, \ldots, M\}$ defined by $\rho_{s}(m)=\operatorname{rank}\left(f_{m s}\right)$ for $m=1, \ldots, M$ with ties resolved at random. Then it follows that the sample $\left\{\hat{f}_{\rho_{s}(1) s}, \ldots, \hat{f}_{\rho_{s}(M) s}\right\}$ has the same rank order structure as the raw ensemble $\left\{f_{1 s}, \ldots, f_{M s}\right\}$. The ECC ensemble of postprocessed forecast fields is thus given by

$$
\left\{\hat{f}_{\rho_{s}(m) s}\right\}_{s \in \mathcal{S}}, \quad m=1, \ldots, M
$$

Schefzik et al. (2013) discuss and compare several alternative methods to draw the sample from each univariate predictive distribution. We use the quantiles in (5) as it follows from results in Bröcker (2012) that this sample maintains the calibration of the univariate forecasts. 


\subsection{Spatial NGR}

The geostatistical output perturbation (GOP, Gel et al., 2004) approach was originally introduced as an inexpensive substitute of a dynamical ensemble based on a single numerical weather prediction. It dresses the deterministic forecast with a simulated forecast error field according to a spatial random process, thus perturbing the outputs of the NWP models rather than their inputs. We propose a spatial NGR method that adopts the ideas from GOP and combines them with the univariate NGR methods described in Sections 3.1 and 3.2 . The result is a multivariate predictive distribution which generates spatially coherent forecast fields while retaining the univariate NGR marginals. The spatial NGR method can thus also be seen as a fully parametric Gaussian copula approach (Möller et al., 2013; Schefzik et al., 2013).

Denote by $\mathbf{Y}=\left\{y_{s}: s \in \mathcal{S}\right\}$ the vector whose components represent the temperature at each location in $\mathcal{S}$, and by $\mathbf{F}_{m}=\left\{f_{m s}: s \in \mathcal{S}\right\}$ the corresponding weather field forecast by the $m$-th ensemble member. The vector $\boldsymbol{\mu}$ of predictive means obtained by marginal NGR postprocessing is given by

$$
\boldsymbol{\mu}= \begin{cases}a \mathbf{1}+b_{1} \mathbf{F}_{1}+\ldots+b_{M} \mathbf{F}_{M} & \left(\mathrm{NGR}_{+}\right), \\ \overline{\mathbf{Y}}^{\mathcal{T}}+b_{1}\left(\mathbf{F}_{1}-\overline{\mathbf{F}}_{1}^{\mathcal{T}}\right)+\ldots+b_{M}\left(\mathbf{F}_{M}-\overline{\mathbf{F}}_{M}^{\mathcal{T}}\right) & \left(\mathrm{NGR}_{c}\right),\end{cases}
$$

where 1 is a vector of length $|\mathcal{S}|$ with all entries equal to $1, \overline{\mathbf{Y}}^{\mathcal{T}}$ is the average observed temperature vector over the training period $\mathcal{T}$ and $\overline{\mathbf{F}}_{m}^{\mathcal{T}}$ denotes the vector consisting of the average temperature forecast by the $m$-th ensemble member over $\mathcal{T}$, see also (2). Similarly, denote by $\mathbf{D}$ the diagonal matrix of the univariate predictive standard deviations $\sigma_{s}$ with $\sigma_{s}^{2}=c+d S_{s}^{2}$ for $\mathrm{NGR}_{+}$and $\sigma_{s}^{2}=c \xi_{s}^{2}+d S_{s}^{2}$ for $\mathrm{NGR}_{c}$.

The spatial NGR multivariate predictive distribution corresponds to the sum of the bias-corrected forecast mean vector given by (7), a zero-mean random vector with correlated components, and a further zero-mean random vector with uncorrelated components representing small-scale variations that cannot be resolved with the available data. That is,

$$
\mathbf{Y} \mid \mathbf{F}_{1}, \ldots, \mathbf{F}_{M}, \mathcal{D}_{\mathcal{S}}^{\mathcal{T}}=\boldsymbol{\mu}+\mathbf{D} \tilde{\mathbf{E}}
$$

where

$$
\tilde{\mathbf{E}}=\sqrt{1-\theta} \cdot \mathbf{E}_{1}+\sqrt{\theta} \cdot \mathbf{E}_{2}, \quad \theta \in[0,1] .
$$

If all components of $\mathbf{E}_{1}$ and $\mathbf{E}_{2}$ have unit variance, the multiplication with $\mathbf{D}$ scales the components of $\tilde{\mathbf{E}}$ such that their variances match those predicted by the univariate NGR postprocessing methods. In particular, the resulting multivariate model features spatially varying predictive variances.

For the spatial correlations we follow Gel et al. (2004) and assume a stationary and isotropic correlation function $C_{\theta, r}$ of the exponential type. That is, we assume that the correlation between two components of $\tilde{\mathbf{E}}$ corresponding to locations $s_{i}$ and $s_{j}$ depends only on their Euclidean distance $\left\|s_{i}-s_{j}\right\|$ and is given by

$$
C_{\theta, r}\left(s_{i}, s_{j}\right)=(1-\theta) \cdot e^{-\frac{\left\|s_{i}-s_{j}\right\|}{r}}+\theta \cdot \delta_{i j},
$$

where $\delta_{i j}$ denotes to the Kronecker delta function which is equal to 1 if $i=j$ and 0 otherwise. The parameter $\theta \in[0,1]$ has already been introduced above and controls the relative contribution of the spatially correlated random vector $\mathbf{E}_{1}$ and the spatially uncorrelated random vector $\mathbf{E}_{2}$ to the overall variance. The range parameter $r>0$ determines the rate at which the spatial correlations of $\mathbf{E}_{1}$ decay with distance. Once those parameters have been estimated, samples of $\tilde{\mathbf{E}}$ can be simulated, scaled by the site specific standard deviations, and added to the forecast mean vector $\boldsymbol{\mu}$.

The resulting spatial NGR multivariate predictive distribution at locations within $\mathcal{S}$ is given by

$$
\mathbf{Y} \mid \mathbf{F}_{1}, \ldots, \mathbf{F}_{M}, \mathcal{D}_{\mathcal{S}} \sim \mathcal{N}_{|\mathcal{S}|}(\boldsymbol{\mu}, \boldsymbol{\Sigma}), \quad \text { with } \boldsymbol{\Sigma}=\mathbf{D P D}
$$

where $\mathcal{N}_{|\mathcal{S}|}$ denotes a multivariate normal distribution of dimension $|\mathcal{S}|$, and $\mathbf{P}$ is the correlation matrix of $\tilde{\mathbf{E}}$. Note that this definition can be extended to locations outside the set $\mathcal{S}$; as pointed out above, both $\boldsymbol{\mu}$ and 
D can be defined for any set of locations where ensemble forecasts are available, and the same is also true for $\mathbf{P}$ since (8) presents a well-defined correlation function over the entire Euclidean plane.

\subsection{Estimating the spatial NGR correlation parameters}

In order to estimate the correlation parameters $\theta$ and $r$ in (8), we consider the standardized forecast errors $\tilde{e}_{s t}:=\left(y_{s t}-\mu_{s t}\right) / \sigma_{s t}$ at all locations $s \in \mathcal{S}$ and on all training days $t \in \mathcal{T}$, and study their average half squared differences over $\mathcal{T}$,

$$
\frac{1}{2|\mathcal{T}|} \sum_{t \in \mathcal{T}}\left(\tilde{e}_{s_{i} t}-\tilde{e}_{s_{j} t}\right)^{2}, \quad s_{i}, s_{j} \in \mathcal{S} .
$$

This way we obtain an empirical version of the variogram

$$
\gamma_{\theta, r}\left(s_{i}, s_{j}\right):=\frac{1}{2} \operatorname{Var}\left(\tilde{\mathbf{E}}_{s_{i}}-\tilde{\mathbf{E}}_{s_{j}}\right)=(1-\theta)\left(1-e^{-\frac{\left\|s_{i}-s_{j}\right\|}{r}}\right)+\theta\left(1-\delta_{i j}\right),
$$

that corresponds to our correlation model $C_{\theta, r}$.

The assumption of a stationary and isotropic correlation function implies that $\gamma_{\theta, r}\left(s_{i}, s_{j}\right)$ is a function of the distance $d\left(s_{i}, s_{j}\right)=\left\|s_{i}-s_{j}\right\|$ only, and so the average half squared differences in (10) can be further aggregated which reduces their variability. Specifically, we sort all pairs $s_{i}, s_{j}$ by their distance into bins $B_{1}, \ldots, B_{L}$ with the bin sizes chosen such that the number of pairs in each bin is approximately equal. The values in (10) are then averaged over each bin, resulting in pairs $\left(d_{l}, \hat{\gamma}_{l}\right)_{l=1, \ldots, L}$ of distances associated with each bin and empirical variogram values that may be compared to the theoretical variogram (11) for given values of $\theta$ and $r$. For the calculation of these values, we employ the R package RandomFields by Schlather (2011). When fitting a curve to the empirical variogram, we follow Berrocal et al. (2007) and use weighted least squares fitting as proposed by Cressie (1985), minimizing the function

$$
S(\theta, r)=\sum_{l=1}^{L} n_{l}\left(\frac{\hat{\gamma}_{l}-\gamma_{\theta, r}\left(d_{l}\right)}{\gamma_{\theta, r}\left(d_{l}\right)}\right)^{2},
$$

where $n_{l}$ is the number of location pairs associated with $B_{l}$. The minimization problem has to be solved numerically for which we use the optimization algorithm by Bryd et al. (1995) as implemented in the Rfunction optim (R Core Team, 2013). The range parameter $r$ is constrained to be positive and not larger than the maximum distance over the entire domain, which equals $890 \mathrm{~km}$. The starting values are kept fixed at the averaged values over the entire forecasting period obtained in earlier experiments.

Alternatively, the random field parameters could be estimated via maximum likelihood. Under ideal conditions, this is statistically more efficient. However, as maximum likelihood estimation is potentially more sensitive to outliers and computationally more expensive we employ the variogram based approach in line with Berrocal et al. (2007). Indeed, results obtained with maximum likelihood estimation (not shown here) slightly reduced the predictive performance of the spatial NGR forecasting methods.

\section{Results}

In this section we present the results of applying the univariate $\mathrm{NGR}_{+}$and $\mathrm{NGR}_{\mathrm{c}}$ postprocessing methods as well as their spatial extensions to forecasts from the COSME-DE ensemble prediction system, described in Section 2. Additionally, we provide a comparison to the univariate ensemble BMA method of Raftery et al. (2005) and the multivariate spatial BMA approach, proposed by Berrocal et al. (2007). The forecast evaluation methods are discussed in the Appendix. 


\subsection{Ensemble BMA reference methods}

The univariate ensemble BMA method of Raftery et al. (2005) is a kernel dressing approach where, for temperature, each bias corrected ensemble member is dressed with a Gaussian kernel, where the variance is kept fixed. That is,

$$
y_{s} \mid f_{m s} \sim \mathcal{N}\left(a_{m}+b_{m} f_{m s}, \sigma^{2}\right),
$$

for $m=1, \ldots, M$ and $s \in \mathcal{S}$. The predictive density is then given by a weighted average

$$
\sum_{m=1}^{M} \omega_{m} \varphi\left(a_{m}+b_{m} f_{m s}, \sigma^{2}\right),
$$

where $\varphi$ denotes the Gaussian density and the weights $\omega_{1}, \ldots, \omega_{M}$ are nonnegative with $\sum_{m=1}^{M} \omega_{m}=1$. The weights reflect the skill of each ensemble member in the training period $\mathcal{T}$. We estimate the ensemble BMA parameters using the $\mathrm{R}$ package ensembleBMA employing the same training period $\mathcal{T}$ as for the univariate NGR methods.

The spatial BMA approach of Berrocal et al. (2007) combines ensemble BMA with the GOP method of Gel et al. (2004) in a similar way as the spatial NGR methods, described in Section 4.2 above. However, it differs from spatial NGR in the manner in which realizations of the multivariate predictive distribution are simulated. For a temperature forecast field under spatial BMA, we first randomly choose a member of the dynamical ensemble according to the ensemble BMA weights in (12), and then dress the corresponding bias-corrected forecast field with an error field that has a stationary covariance structure specific to this member. As the forecast field is chosen at random and the covariance function is member-specific, the final covariance structure becomes non-stationary. This comes at the expense of having to estimate $M$ different covariance functions, while spatial NGR requires a single correlation function only, and achieves spatially varying variances through scaling.

\subsection{Univariate predictive performance}

Measures of univariate predictive performance of the raw COSMO-DE ensemble and the postprocessed forecasts under $\mathrm{NGR}_{+}, \mathrm{NGR}_{\mathrm{c}}$, and $\mathrm{BMA}$ are given in Table 1 . A simple approach to assess calibration and sharpness of univariate probabilistic forecasts is to calculate the nominal coverage and width of prediction intervals. The $90.5 \%$ prediction interval considered here corresponds to the probability that the observation is within the ensemble range, assuming that the ensemble members and the observation are exchangeable. While the raw ensemble returns very sharp forecasts, it is severely underdispersive as can be seen by the insufficient coverage. This is also reflected in the numerical scores which are significantly better for all three postprocessing methods. $\mathrm{NGR}_{+}$and ensemble BMA return essentially identical scores, improving upon the ensemble by $34 \%$ in terms of the CRPS and by approximately $18 \%$ in terms of both mean absolute error (MAE) and root mean squared error (RMSE). Ensemble BMA returns minimally wider prediction intervals than $\mathrm{NGR}_{+}$but yields an empirical coverage that is closest to the nominal $90.5 \%$. The locally adaptive postprocessing of $\mathrm{NGR}_{\mathrm{c}}$ yields the best overall scores and approximately $10 \%$ shorter prediction intervals than $\mathrm{NGR}_{+}$on average despite being slightly underdispersive. The station-specific reliability indices indicate that the postprocessing improves the calibration consistently across the country with the postprocessing methods always yielding lower indicies than the average of the ensemble.

\subsection{Spatial calibration}

In Figure 3 we assess the calibration of the joint forecast fields at all 514 observation stations in Germany using multivariate band depth rank histograms (see the Appendix for details). Without additional spatial 
Table 1: Mean continuous ranked probability score (CRPS), mean absolute error (MAE) and root mean squared error (RMSE) for $21 \mathrm{~h}$ temperature forecasts aggregated over all 514 stations and 346 days in the test set. Also reported here are the average width (PI-W) and coverage (PI-C) of $90.5 \%$ prediction intervals aggregated over the entire test set and the mean (RI-Mean), minimum (RI-Min) and maximum (RI-Max) station-specific reliability indicies.

\begin{tabular}{lcccccccc}
\hline & $\begin{array}{c}\text { CRPS } \\
\left({ }^{\circ} \mathrm{C}\right)\end{array}$ & $\begin{array}{c}\text { MAE } \\
\left({ }^{\circ} \mathrm{C}\right)\end{array}$ & $\begin{array}{c}\text { RMSE } \\
\left({ }^{\circ} \mathrm{C}\right)\end{array}$ & $\begin{array}{c}\text { PI-W } \\
\left({ }^{\circ} \mathrm{C}\right)\end{array}$ & $\begin{array}{c}\text { PI-C } \\
(\%)\end{array}$ & RI-Mean & RI-Min & RI-Max \\
\hline Raw ensemble & 1.56 & 1.77 & 2.27 & 1.50 & 26.0 & 1.29 & 0.72 & 1.63 \\
$\mathrm{BMA}$ & 1.04 & 1.46 & 1.86 & 5.91 & 88.9 & 0.35 & 0.14 & 0.97 \\
$\mathrm{NGR}_{+}$ & 1.04 & 1.46 & 1.87 & 5.76 & 88.0 & 0.35 & 0.12 & 0.93 \\
$\mathrm{NGR}_{\mathrm{c}}$ & 0.96 & 1.35 & 1.73 & 5.17 & 86.6 & 0.23 & 0.13 & 0.84 \\
\hline
\end{tabular}

modeling (i.e. assuming independent forecast errors at the different stations) the multivariate calibration of BMA, NGR + , and $\mathrm{NGR}_{\mathrm{c}}$ is rather poor, despite their good marginal calibration. The three spatial forecasts that are based on parametric modeling of the error field - spatial BMA, spatial NGR + and spatial NGR $\mathrm{N}_{\mathrm{c}}-$ significantly improve upon the calibration of the univariate methods, in particular spatial $\mathrm{NGR}_{\mathrm{c}}$. However, the strength of the correlations seems somewhat too low as the observed field is too often either the most central or the most outlying field resulting in a $\cup$-shaped histogram (see also Section 4 of Thorarinsdottir et al. 2013). In contrast, the combination of ECC and NGR produces forecasts fields where the strength of the correlations appears slightly too high. This result is supported by the spatial correlation patterns portrayed in Figure 1 where the raw ensemble - and thus also the ECC fields - appears to have significantly longer-range correlations than the estimated Gaussian error fields.

In our spatial $\mathrm{NGR}_{+} / \mathrm{NGR}_{\mathrm{c}}$ model we made the simplifying assumption of a stationary and isotropic correlation function. In order to check whether this assumption is appropriate or whether correlation strengths vary strongly over the domain considered here, we study the probability integral transforms (PITs) of predicted temperature differences between close-by stations (a motivation and interpretation for that is given in the Appendix). Specifically, we focus on the $\mathrm{NGR}_{\mathrm{c}}$ model and its spatial extensions where we can assume that the univariate predictive distributions have no local biases and reflect the local prediction uncertainty reasonably well (Scheuerer and König, 2014). Figure 4 depicts, for each station, the mean absolute deviations of the PIT values from 0.5 over all verification days and all temperature differences between this station and stations within a $50 \mathrm{~km}$ neighborhood. As expected, in the absence of a spatial model the magnitude of temperature differences is overestimated. When ECC is used to restore the rank correlations of the raw ensemble, it is underestimated (i.e. spatial correlations are too strong), which is in line with our conclusions from Figure 3. On average, the mean absolute deviations from 0.5 of the PIT values corresponding to spatial $\mathrm{NGR}_{\mathrm{c}}$ are closest to the value 0.25 that corresponds to perfect calibration. However, the adequate correlation strength varies across the domain. The assumption of stationarity and isotropy of our statistical correlation model entails too weak correlations over the North German Plain and too strong correlations near the Alpine foothills and in the vicinity of the various low mountain ranges. After all, (8) presents a good first approximation, but a more sophisticated, non-stationary correlation model may yield further improvement.

\subsection{Case study I: Predictive performance in Saarland}

For a more quantitative assessment of multivariate predictive performance, we focus on two smaller subsets of the 514 stations. This is necessary because in our own experience, the lack of sensitivity of the energy score to misspecifications of the spatial correlation structure (Pinson and Tastu, 2013) becomes even worse as the dimension of locations considered simultaneously increases.

First, we consider the joint predictive distribution at the seven stations in the state of Saarland (see 
BMA

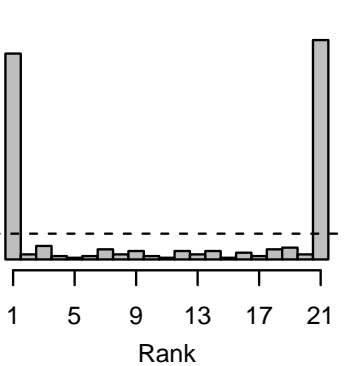

Spatial BMA
NGR+

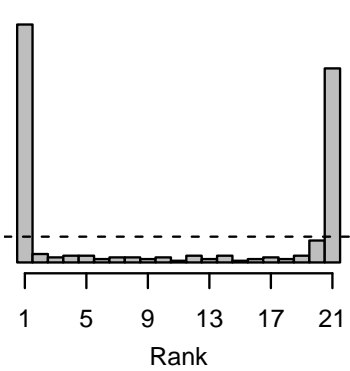

Spatial NGR+
NGRc

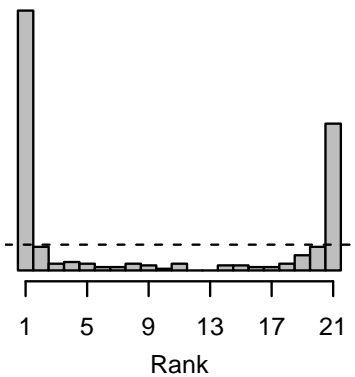

Spatial NGRc
ECC NGR+

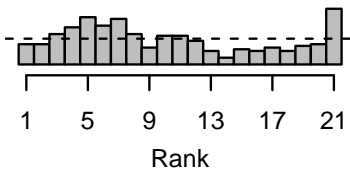

ECC NGRC
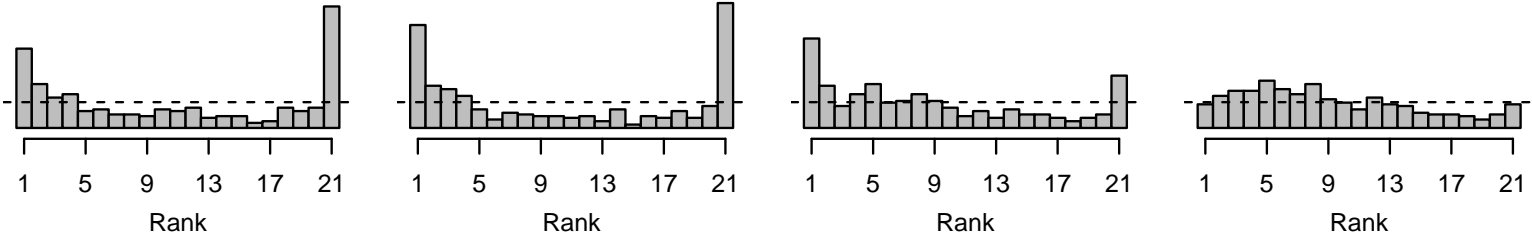

Figure 3: Multivariate band depth rank histograms to assess the calibration of joint forecast fields at 514 stations in Germany aggregated over the 346 days in the test set.
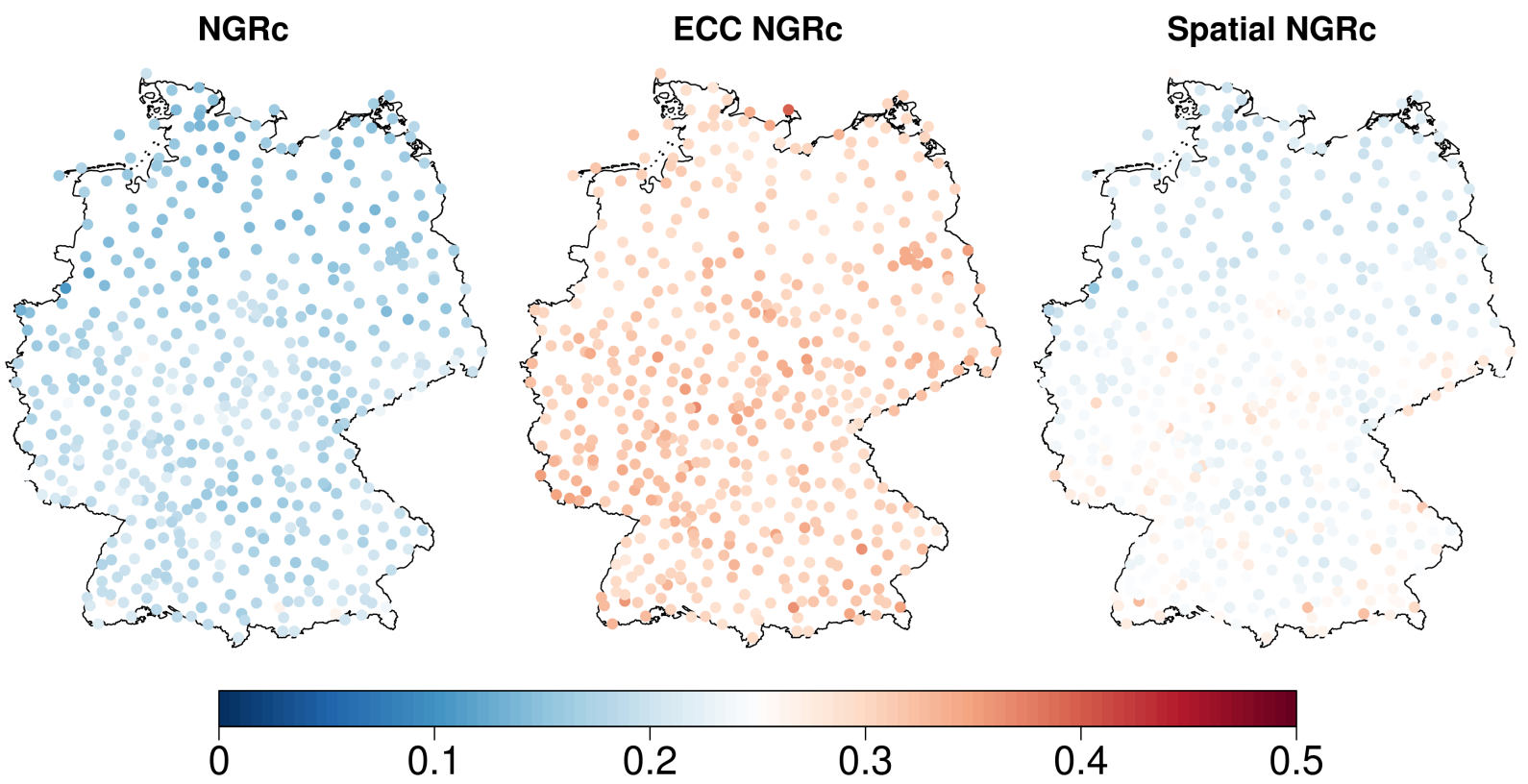

Figure 4: Mean absolute deviations of the temperature difference PIT values from 0.5 where the mean is taken over all verification days and, for each station, over all stations within a radius of $50 \mathrm{~km}$. 
BMA

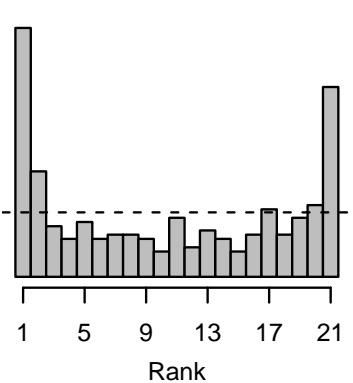

Spatial BMA

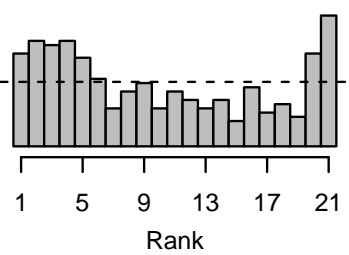

NGR+

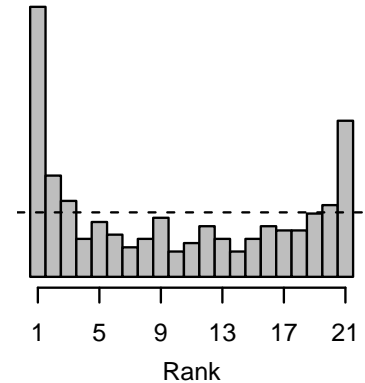

Spatial NGR+

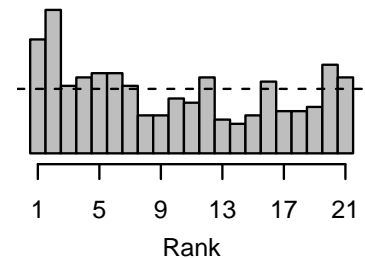

NGRc

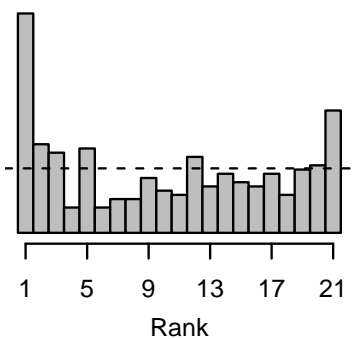

Spatial NGRc

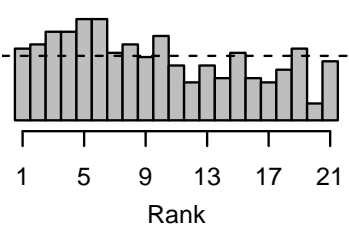

ECC NGR+

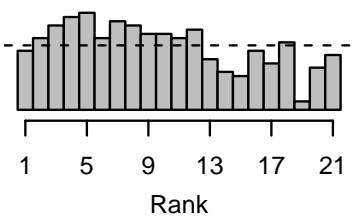

ECC NGRC

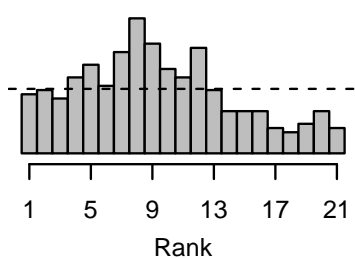

Figure 5: Multivariate band depth rank histograms to assess the calibration of joint temperature forecasts at the seven observation stations in the state of Saarland in Germany aggregated over the 346 days in the test set. 
Table 2: Average energy score (ES), Euclidean error (EE) and Dawid-Sebastiani score (DS) of joint temperature forecasts at seven observation stations in the state of Saarland in Germany over all 346 days in the test set.

\begin{tabular}{lcrr}
\hline & $\begin{array}{c}\text { ES } \\
\left({ }^{\circ} \mathrm{C}\right)\end{array}$ & $\begin{array}{c}\mathrm{EE} \\
\left({ }^{\circ} \mathrm{C}\right)\end{array}$ & \multicolumn{1}{c}{$\mathrm{DS}$} \\
\hline Raw ensemble & 5.27 & 5.86 & 21210.9 \\
$\mathrm{BMA}$ & 3.59 & 4.90 & 20.6 \\
NGR $_{+}$ & 3.59 & 4.91 & 20.7 \\
NGR $_{\mathrm{c}}$ & 3.28 & 4.55 & 17.4 \\
Spatial BMA & 3.56 & 4.90 & 16.8 \\
Spatial NGR $_{+}$ & 3.57 & 4.91 & 16.9 \\
Spatial NGR $_{\mathrm{c}}$ & 3.25 & 4.55 & 14.2 \\
ECC NGR $_{+}$ & 3.69 & 4.92 & 1112.9 \\
ECC NGR $_{\mathrm{c}}$ & 3.36 & 4.55 & 1665.1 \\
\hline
\end{tabular}

Figure 22. The corresponding multivariate band depth rank histograms in Figure 5 confirm the conclusions from the preceding subsection in that spatial modeling significantly improves the joint calibration of the standard (non-spatial) postprocessing methods. However, the slight bump at the left side of the histograms for spatial $\mathrm{NGR}_{\mathrm{c}}, \mathrm{ECC} \mathrm{NGR}_{+}$and $\mathrm{ECC} \mathrm{NGR}_{\mathrm{c}}$ suggest that for this specific region, the correlation under these models is slightly too high over longer distances. The opposite holds for spatial BMA and spatial NGR where the observed field disproportionally often takes either the lowest or the highest rank. Overall, the multivariate methods still yield histograms which are much closer to uniformity than the univariate methods.

Table 2 shows the multivariate scores over this region, and while these results are subject to some sampling variability, they show again a clear tendency of the spatial models yielding better multivariate performance in respect to their univariate counterparts with spatial $\mathrm{NGR}_{\mathrm{c}}$ being especially competitive. The somewhat counter-intuitive result that the energy scores of ECC NGR $\mathrm{N}_{\mathrm{c}}$ and ECC NGR + are larger than those of $\mathrm{NGR}_{\mathrm{c}}$ and $\mathrm{NGR}_{+}$might be explained by sampling effects. While the two latter are based on 10,000 samples, the ECC ensembles and the raw ensemble consist of only 20 members. This does not warrant a stable estimation of the empirical covariance matrix which can be disastrous when calculating the DawidSebastiani score and also have a negative impact on the energy score. On the other hand, it illustrates that it can be problematic in certain contexts that ECC NGR + and ECC NGR ${ }_{c}$ inherit the sometimes close to singular correlation matrices from the raw COSMO-DE ensemble forecasts.

\subsection{Case study II: Minimum temperature along the highway A3}

As a second example in which the multivariate aspect of the predictive distributions becomes noticeable, we consider the task of predicting the minimum temperature along a section of the highway A3 which connects the two cities Frankfurt am Main and Cologne. For consistency with the forecasts at the individual stations and with other composite quantities, we do not set up a separate postprocessing model for minimum temperature, but derive it by taking the minimum over 11 stations along this section of the A3.

Since the minimum of several random variables depends not only on their means and variances, but also on their correlations, we expect that only the spatial postprocessing methods can provide reasonably calibrated probabilistic forecasts. Indeed, the histograms in Figure 6 show that without spatial modeling the minimum temperature is systematically underestimated. This is a consequence of the fact that the minimum over independent random variables is on average much smaller than the minimum over positively correlated random variables. This systematic underestimation is largely avoided by spatial BMA, spatial $\mathrm{NGR}_{+}$and spatial $\mathrm{NGR}_{\mathrm{c}}$ while the ECC techniques here yield the histograms closest to uniformity. This clear advantage 


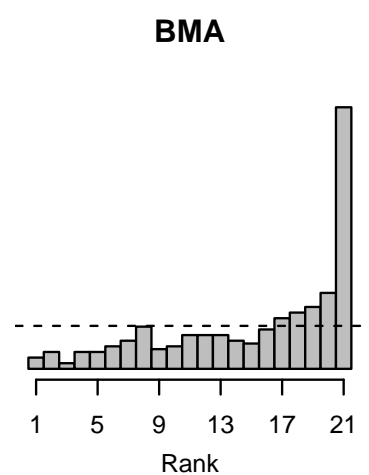

Spatial BMA
NGR+

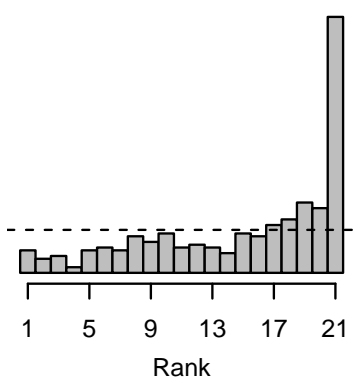

Spatial NGR+
NGRc

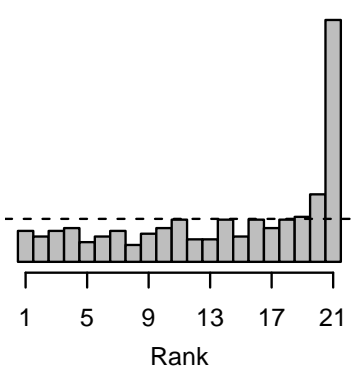

Spatial NGRc
ECC NGR+

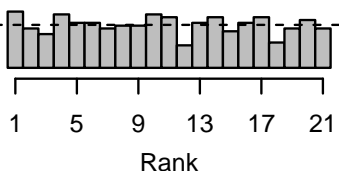

ECC NGRc
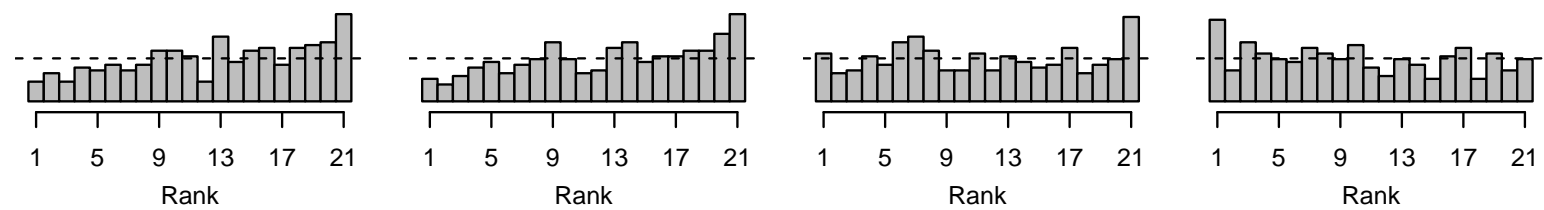

Figure 6: Verification rank histograms for forecasts of the minimum temperature over 11 stations along a section of the highway A3, where the different cases correspond to the days of the verification period. 
Table 3: CRPS and MAE for minimum temperature forecasts over 11 stations along the highway A3 averaged over all verification days. The last column gives the BS for the event that the temperature drops below freezing $\left(0^{\circ} \mathrm{C}\right)$ at at least one of these stations. The average is calculated over the winter subset of verification days in January, February and November 2011.

\begin{tabular}{lccc}
\hline & $\begin{array}{c}\text { CRPS } \\
\left({ }^{\circ} \mathrm{C}\right)\end{array}$ & $\begin{array}{c}\text { MAE } \\
\left({ }^{\circ} \mathrm{C}\right)\end{array}$ & $\mathrm{BS}_{0}$ \\
\hline Raw ensemble & 1.74 & 1.92 & 0.120 \\
BMA & 1.08 & 1.41 & 0.121 \\
NGR $_{+}$ & 1.05 & 1.37 & 0.114 \\
NGR $_{\mathrm{c}}$ & 0.96 & 1.27 & 0.103 \\
Spatial BMA & 0.86 & 1.20 & 0.079 \\
Spatial NGR $_{+}$ & 0.86 & 1.20 & 0.082 \\
Spatial NGR $_{\mathrm{c}}$ & 0.82 & 1.16 & 0.079 \\
ECC NGR $_{+}$ & 0.84 & 1.18 & 0.085 \\
ECC NGR $_{\mathrm{c}}$ & 0.84 & 1.17 & 0.083 \\
\hline
\end{tabular}

of postprocessing methods that account for spatial correlations is further confirmed by the CRPS and MAE scores in Table 3 ,

As an application and example of the relevance of spatial modeling in practice, consider the decision problem of dispatching or not dispatching salt spreaders when the temperatures along the considered section of the $\mathrm{A} 3$ are predicted to fall below $0^{\circ} \mathrm{C}$. The event "temperature falls below $0^{\circ} \mathrm{C}$ at at least one location along the $\mathrm{A} 3$ " is equivalent to "minimum temperature along the $\mathrm{A} 3$ falls below $0{ }^{\circ} \mathrm{C}$ ", and good decisions are therefore taken if this event is predicted accurately. The last column of Table 3 shows the corresponding average Brier scores (BS) over the verification days in the winter months of January, February and November, and illustrates once again that appropriate consideration of spatial dependence is required to take full advantage of statistical postprocessing.

\section{Discussion}

In this paper we have proposed a postprocessing method for temperature that uses the information of a dynamical ensemble as inputs and generates a calibrated statistical ensemble as an output. In doing so, it not only yields calibrated marginal predictive distributions but entire temperature forecast fields, thus aiming for multivariate calibration. The importance of this property is underlined by the results presented in Section 5 where forecasts of spatially aggregated quantities are studied and spatial correlations have to be considered. Our spatial $\mathrm{NGR}_{+}$approach performs similar to the spatial BMA approach of Berrocal et al. (2007). However, it is conceptually simpler and computationally more efficient; the estimation of the spatial correlation structure of spatial BMA is $M$ times more expensive than that of spatial $\mathrm{NGR}_{+}$, where $M$ is the size of the original ensemble. This makes it an attractive alternative, especially since further extensions such as the spatial $\mathrm{NGR}_{\mathrm{c}}$ method presented here - are also easier to implement.

In our case study using the ensemble forecasts of the COSMO-DE-EPS, the performance of the parametric spatial methods was overall slightly better than the results obtained by modeling spatial dependence via ECC. This result does not hold in any case. When the (spatial) correlation structure of the ensemble represents the true multivariate uncertainty well, methods that use or retain the rank correlations (Roulin and Vannitsem, 2011; Schefzik et al., 2013, Van Schaeybroeck and Vannitsem, 2013) have the potential advantage that they can feature flow-dependent dependence structures while the statistical models presented here 
rely on the assumption that correlations are constant over a certain period of time. A statistical approach, on the other hand, has the advantage that it determines the correlation structure based on both forecasts and observations, and thus does not inherit (or even amplify) spurious and wrong correlations that may be present in the ensemble.

The exponential correlation function used by Gel et al. (2004), Berrocal et al. (2007), and in the present paper is of course a somewhat simplistic model. While replacing it by a function from the more general Matérn class, which nests the exponential model as a special case, did not improve the performance of our method, Figure 4 suggests that a non-stationary correlation function might yield a better approximation of the true spatial dependence structure. There are a number of non-parametric modeling approaches that can potentially deal with these kinds of effects (Anderes, 2011; Lindgren et al., 2011; Jun et al., 2011, Kleiber et al., 2013). However, this is rather challenging and left for future research. A further extension of the approach presented here concerns correlations between different lead times. Instead of modeling spatial correlations only one would need to set up a model that captures correlations in both space and time. Similarly, some applications require appropriate correlations between different weather variables. This presents yet another multivariate aspect that has been addressed by Möller et al. (2013). Taking all three aspects - space, time, and different variables - into account would be the ultimate goal in multivariate modeling. At the same time, this further increases the level of complexity so that in this very general setting the ECC approach might be preferred just for the sake of simplicity.

\section{Acknowledgments}

The authors thank Tilmann Gneiting for sharing his thoughts and expertise. This work was funded by the German Federal Ministry of Education and Research, within the framework of the extramural research program of Deutscher Wetterdienst and by Statistics for Innovation, $\mathrm{sfi}^{2}$ in Oslo, Norway.

\section{References}

Anderes, E. and Stein, M. L. (2011). Local likelihood estimation for nonstationary random fields. J Multivariate Anal 102, 506-520.

Anderson, J. L. (1996). A method for producing and evaluating probabilistic forecasts from ensemble model integrations. J Climate 9(7), 1518-1530.

Baldauf, M., A. Seifert, J. Förstner, D. Majewski, M. Raschendorfer, and T. Reinhardt (2011). Operational convective-scale numerical weather prediction with the COSMO model: description and sensitivities. Mon Weather Rev 139(12), 3887-3905.

Berrocal, V. J., A. E. Raftery, and T. Gneiting (2007). Combining spatial statistical and ensemble information in probabilistic weather forecasts. Mon Weather Rev 135(4), 1386-1402.

Brier, G. W. (1950). Verification of forecasts expressed in terms of probability. Mon Weather Rev 78, 1-3.

Bröcker, J. (2012). Evaluating raw ensembles with the continous ranked probability score. Q J Roy Meteor Soc 138, 1611-1617.

Bröcker, J. and L. A. Smith (2008). From ensemble forecasts to predictive distribution functions. Tellus A 60, 663-678.

Bryd, R. H., P. Lu, J. Nocedal, and C. Zhu (1995). A limited memory algorithm for bound constrained optimization. SIAM J Sci Comp 16, 1190-1208. 
Cressie, N. A. C. (1985). Fitting variogram models by weighted least squares. Math Geol 17, 563-586.

Dawid, A. P. (1984). Statistical theory: The prequential approach (with discussion and rejoinder). J Roy Stat Soc A 147, 278-292.

Dawid, A. P. and P. Sebastiani (1999). Coherent dispersion criteria for optimal experimental design. Ann Stat 27, 65-81.

Delle Monache, L., J. P. Hacker, Z. Y., D. X., and S. R. B. (2006). Probabilistic aspects of meteorological and ozone regional ensemble forecasts. J Geophys Res 111, D24307.

Doms, G. and U. Schättler (2002). A description of the nonhydrostatic regional model LM : Dynamics and numerics. Technical report, Deutscher Wetterdienst.

Gebhardt, C., S. E. Theis, M. Paulat, and Z. Ben-Bouallègue (2011). Uncertainties in COSMO-DE precipitation forecasts introduced by model perturbations and variation of lateral boundaries. Atmos Res 100, $168-177$.

Gel, Y., A. E. Raftery, and T. Gneiting (2004). Calibrated probabilistic mesoscale weather field forecasting: The geostatistical output perturbation (GOP) method (with discussion and rejoinder). J Am Stat Assoc 99, $575-590$.

Gneiting, T. (2011). Making and evaluating point forecasts. J Am Stat Assoc 106(494), 746-762.

Gneiting, T., F. Balabdaoui, and A. E. Raftery (2007). Probabilistic forecasts, calibration and sharpness. $J$ Roy Stat Soc B 69(2), 243-268.

Gneiting, T. and A. E. Raftery (2007). Strictly proper scoring rules, prediction, and estimation. J Am Stat Assoc 102(477), 359-378.

Gneiting, T., A. E. Raftery, A. H. Westveld, and T. Goldman (2005). Calibrated probabilistic forecasting using ensemble model output statistics and minimum CRPS estimation. Mon Weather Rev 133(5), 10981118.

Grimit, E. P., T. Gneiting, V. J. Berrocal, and N. A. Johnson (2006). The continuous ranked probability score for circular variables and its application to mesoscale forecast ensemble verification. $Q J$ Roy Meteor Soc 132, 2925-2942.

Hagedorn, R., T. M. Hamill, and J. S. Whitaker (2008). Probabilistic forecast calibration using ECMWF and GFS ensemble reforecasts. Part I: Two-meter temperatures. Mon Weather Rev 136, 2608-2619.

Hamill, T. M. and S. J. Colucci (1997). Verification of Eta-RSM Short-Range Ensemble Forecasts. Mon Weather Rev 125(6), 1312-1327.

Jun, M., I. Szunyogh, M. G. Genton, F. Zhang, and C. H. Bishop (2011). A statistical investigation of the sensitivity of ensemble-based Kalman filters to covariance filtering. Mon Weather Rev 139(9), 3036-3051.

Kann, A., C. Wittmann, Y. Wang, and X. Ma (2009). Calibrating 2-m temperature of limited-area ensemble forecasts using high-resolution analysis. Mon Weather Rev 137, 3373-3387.

Kleiber, W., R. Katz, and B. Rajagopalan (2013). Daily minimum and maximum temperature simulation over complex terrain. Ann Appl Stat 7, 588-612. 
Kleiber, W., A. E. Raftery, J. Baars, T. Gneiting, C. F. Mass, and E. P. Grimit (2011). Locally calibrated probabilistic temperature forecasting using geostatistical model averaging and local Bayesian model averaging. Mon Weather Rev 139(570), 2630-2649.

Lerch, S. and T. L. Thorarinsdottir (2013). Comparison of nonhomogeneous regression models for probabilistic wind speed forecasting. Tellus A 65, 21206.

Leutbecher, M. and T. N. Palmer (2008). Ensemble forecasting. J Comput Phys 227, 3515-3539.

Lewis, J. M. (2005). Roots of Ensemble Forecasting. Mon Weather Rev 133, 1865-1885.

Lindgren, F., H. Rue, and J. Lindström (2011). An explicit link between Gaussian fields and Gaussian Markov random fields: The stochastic partial differential equation approach" (with discussion). J Roy Stat Soc B 73, 423-498.

Möller, A., A. Lenkoski, and T. L. Thorarinsdottir (2013). Multivariate probabilistic forecasting using Bayesian model averaging and copulas. Q J Roy Meteor Soc 139(673), 982-991.

Peralta, C. and M. Buchhold (2011). Initial condition perturbations for the COSMO-DE-EPS. COSMO Newsletter 11, 115-123.

Pinson, P. and J. Tastu (2013). Discrimination ability of the energy score. Technical report, Technical University of Denmark.

R Core Team (2013). R: A Language and Environment for Statistical Computing. Vienna, Austria: R Foundation for Statistical Computing.

Raftery, A. E., T. Gneiting, F. Balabdaoui, and M. Polakowski (2005). Using Bayesian model averaging to calibrate forecast ensembles. Mon Weather Rev 133(5), 1155-1174.

Rasmussen, C. E. and C. K. I. Williams (2006). Gaussian processes for machine learning. Cambridge, MA: The MIT Press.

Roulin, E. and S. Vannitsem (2011). Post-processing of ensemble precipitation predictions with extended logistic regression based on hindcasts. Mon Weather Rev 47, 874-888.

Schefzik, R., T. L. Thorarinsdottir, and T. Gneiting (2013). Uncertainty quantification in complex simulation models using ensemble copula coupling. Stat Sci 28(4), 616-640.

Scheuerer, M. (2014). Probabilistic quantitative precipitation forecasting using ensemble model output statistics. Q J Roy Meteor Soc 140, 1086-1096.

Scheuerer, M. and L. Büermann (2014). Spatially adaptive post-processing of ensemble forecasts for temperature. J Roy Stat Soc C 63, 405-422.

Scheuerer, M. and G. König (2014). Gridded locally calibrated, probabilistic temperature forecasts based on ensemble model output statistics. Q J Roy Meteor Soc. To appear. DOI:10.1002/qj.2323.

Schlather, M. (2011). RandomFields: Simulation and Analysis of Random Fields.

Steppeler, J., G. Doms, U. Schättler, H. W. Bitzer, A. Gassmann, U. Damrath, and G. Gregoric (2003). Meso-gamma scale forecasts using the nonhydrostatic model LM. Meteorol Atmos Phys 82, 75-96.

Thorarinsdottir, T. L. and T. Gneiting (2010). Probabilistic forecasts of wind speed: Ensemble model output statistics by using heteroscedastic censored regression. J Roy Stat Soc A 173(2), 371-388. 
Thorarinsdottir, T. L. and M. S. Johnson (2012). Probabilistic wind gust forecasting using non-homogeneous gaussian regression. Mon Weather Rev 140, 889-897.

Thorarinsdottir, T. L., M. Scheuerer, and C. Heinz (2013). Assessing the calibration of high-dimensional ensemble forecasts using rank histograms. arXiv:1310.0236.

Van Schaeybroeck, B. and S. Vannitsem (2013). Ensemble post-processing using member-by-member approaches. In submission.

Wilks, D. S. (2011). Statistical Methods in the Atmospheric Sciences (3rd ed.). Elsevier Academic Press, Amsterdam.

Wilks, D. S. and T. M. Hamill (2007). Comparison of ensemble-MOS methods using GFS reforecasts. Mon Weather Rev 135, 2379-2390.

\section{A Forecast evaluation methods}

Statistical postprocessing aims at correcting systematic biases and/or misrepresentation of the forecast uncertainty in the raw ensemble and, in our case, returns full probabilistic distributions. To evaluate the predictive performance of the methods under consideration, we follow Gneiting et al. (2007) who state that the goal of probabilistic forecasting is to maximize the sharpness of the predictive distribution subject to calibration.

\section{A.1 Assessing calibration}

Calibration refers to the statistical compatibility between the forecasts and the observations; the forecast is calibrated if the observation cannot be distinguished from a random draw from the predictive distribution. For continuous univariate distributions, calibration can be assessed empirically by plotting the histogram of the probability integral transform (PIT) - the value of the predictive cumulative distribution function in the observed value (Dawid, 1984, Gneiting et al., 2007) - over all forecast cases. A forecasting method that is calibrated on average will return a uniform histogram, a $\cap$-shape indicates overdispersion and a $\cup$ shape indicates underdispersion while a systematic bias results in a triangular shape histogram. The discrete equivalent of the PIT histogram, which applies to ensemble forecasts, is the verification rank histogram (Anderson, 1996; Hamill and Colucci, 1997). It shows the distribution of the ranks of the observations within the corresponding ensembles and has the same interpretation as the PIT histogram. In order to facilitate direct comparison of the various methods, we only employ the rank histogram. That is, for the continuous predictive distributions, we create a 20 -member ensemble given by 20 random samples from the distribution.

For multivariate settings, we employ the band depth rank histogram proposed by Thorarinsdottir et al. (2013). This approach ranks the observation within a sample of forecast scenarios by assessing the centrality of the observation within the sample. Let $\mathbf{X}=\left\{\mathbf{x}_{1}, \ldots, \mathbf{x}_{M+1}\right\}=\left\{\mathbf{Y}, \mathbf{F}_{1}, \ldots, \mathbf{F}_{M}\right\}$ denote a set of $M$ forecast vectors and the observation $\mathbf{Y}$, each of dimension $d$. To calculate the band depth rank of the observation $\mathbf{Y}$ in $\mathbf{X}$, we first apply the pre-rank function

$$
\begin{aligned}
r(\mathbf{x}) & =\frac{1}{d} \sum_{k=1}^{d} \sum_{1 \leq i_{1}<i_{2} \leq M+1} \mathbf{1}\left\{\min \left\{x_{i_{1} k}, x_{i_{2} k}\right\} \leq x_{k} \leq \max \left\{x_{i_{1} k}, x_{i_{2} k}\right\}\right\} \\
& =\frac{1}{d} \sum_{k=1}^{d}\left[M+1-\operatorname{rank}_{\mathbf{X}}\left(x_{k}\right)\right]\left[\operatorname{rank}_{\mathbf{X}}\left(x_{k}\right)-1\right]+M,
\end{aligned}
$$


to all vectors $\mathbf{x} \in \mathbf{X}$, where $\mathbf{1}\{\cdot\}$ denotes the indicator function and $\operatorname{rank}_{\mathbf{X}}\left(x_{k}\right)$ denote the rank of element $k$ of the vector $\mathbf{x}$ in $\mathbf{X}$. The band depth rank of $\mathbf{x}_{i}$ is then given by the rank of $r\left(\mathbf{x}_{i}\right)$ in $\left\{r\left(\mathbf{x}_{1}\right), \ldots, r\left(\mathbf{x}_{M+1}\right)\right\}$ with ties resolved at random. Calibrated forecasts should result in a uniform histogram. However, the interpretation of miscalibration in the band depth rank histogram is somewhat different than that of the classic univariate rank histogram. A skew histogram with too many high ranks is an indication of an overdispersive ensemble while to many low ranks can result from either an underdispersive or biased ensemble. Furthermore, too high correlations in the ensemble produce a $\cap$-shaped histogram while a $\cup$-shaped histogram is an indication of a lack of correlation in the ensemble.

Alternatively, we also investigate the fit of the correlation structure by investigating the calibration of predicted temperature differences at close-by stations. Under the multivariate predictive distribution model in (9), the predictive distribution of the temperature difference between location $s_{i}$ and $s_{j}$ is given by

$$
(\Delta y)_{s_{i} s_{j}} \mid f_{1 s_{i}}, f_{1 s_{j}}, \ldots, f_{M s_{i}}, f_{M s_{j}}, \mathcal{D}_{\mathcal{S}}^{\mathcal{T}} \sim \mathcal{N}\left(\mu_{s_{i}}-\mu_{s_{j}}, \sigma_{s_{i}}^{2}-2 \rho_{s_{i} s_{j}} \sigma_{s_{i}} \sigma_{s_{j}}+\sigma_{s_{j}}^{2}\right),
$$

where $\mu_{s_{i}}, \mu_{s_{j}}$ and $\sigma_{s_{i}}^{2}, \sigma_{s_{j}}^{2}$ are the predictive means and variances at $s_{i}$ and $s_{j}$ and $\rho_{s_{i} s_{j}}=C_{\theta, r}\left(s_{i}, s_{j}\right)$ is the correlation between the forecast errors at those two locations. For each station, we calculate the observed temperature differences between this station and all stations within a radius of $50 \mathrm{~km}$, and calculate the PIT values of the predictive distributions given by (13). In the absence of a spatial model, we take $\rho_{s_{i} s_{j}}=0$ for all $s_{i}, s_{j} \in \mathcal{S}$. For a combination of ECC and NGR where the multivariate distribution is represented by an ensemble, we approximate $(13)$ by the empirical CDF of the temperature differences predicted by the individual ensemble members.

Assuming no local biases and marginal calibration, the calibration of the temperature difference forecasts mainly depends on the correct specification of $\rho_{s_{i} s_{j}}$. It will be underdispersive if $\rho_{s_{i} s_{j}}$ is overestimated and overdispersive if $\rho_{s_{i} s_{j}}$ is underestimated. If the strength of spatial correlations implied by the respective postprocessing approach is adequate, the predictive distributions of temperature differences are calibrated and the corresponding PIT values are uniformly distributed on $[0,1]$. Underestimating the correlation strength would entail $\cap$-shaped PIT histograms, i.e. PIT values would tend to accumulate around 0.5. Conversely, overestimating the correlation strength would yield PIT values closer to 0 or 1. A station-specific PIT histogram may thus be summarized by the mean absolute deviations of the PIT values from 0.5 over all verification days and all temperature differences between this station and stations within the $50 \mathrm{~km}$ neighborhood.

The information provided by a rank histogram may also be summarized numerically by the reliability index (RI) which is defined as

$$
\mathrm{RI}=\sum_{i=1}^{I}\left|\zeta_{i}-\frac{1}{I}\right|,
$$

where $I$ is the number of (equally-sized) bins in the histogram and $\zeta_{i}$ is the observed relative frequency in bin $i=1, \ldots, I$. The reliability index thus measures the departure of the rank histogram from uniformity (Delle Monache et al., 2006).

\section{A.2 Scoring rules}

While rank histograms are a useful calibration diagnostic tool, they do not yield information on the sharpness of the predictive distributions. The latter can be evaluated by studying the average width of prediction intervals, which should be as small as possible, provided that the empirical coverage is close to the nominal coverage. As a quantitative measure for predictive performance that takes both calibration and sharpness into account, we employ several proper scoring rules (Gneiting and Raftery, 2007). The different scores assess different aspects of the forecasts. However, they are all negatively oriented in that a smaller score indicates a better forecast. For events with a binary outcome, e.g. "the temperature $y$ does not exceed a certain threshold 
$x "$, we use the Brier score (Brier, 1950)

$$
\operatorname{bs}_{x}(G, y)=\left(\mathbf{1}_{\{y \leq x\}}-G(x)\right)^{2}
$$

where $\mathbf{1}_{\{y \leq x\}}$ is equal to one if $y \leq x$ and zero otherwise, and $G(x)$ is the predicted probability for $y \leq x$.

The continuous ranked probability score (CRPS) in (4) is the integral of the Brier scores over all thresholds $x \in \mathbb{R}$ and thus an overall performance measure. When the integral in (4) is not available in a closed form, the equivalent formulation

$$
\operatorname{crps}(G, y)=\mathbb{E}|X-y|-\frac{1}{2} \mathbb{E}\left|X-X^{\prime}\right|
$$

may be employed instead (Gneiting and Raftery, 2007). Here, $\mathbb{E}$ denotes expectation, $|\cdot|$ stands for the absolute value and $X$ and $X^{\prime}$ are independent copies of a random variable with cumulative distribution function $G$. To estimate the expression in (14), we generate two independent samples $\mathbf{x}=\left\{x_{j}\right\}_{j=1}^{J}$ and $\mathbf{x}^{\prime}=\left\{x_{j}^{\prime}\right\}_{j=1}^{J}$ from the predictive distribution and calculate

$$
\widehat{\operatorname{crps}}(G, y)=\sum_{j=1}^{J}\left|x_{j}-y\right|-\frac{1}{2} \sum_{j=1}^{J}\left|x_{j}-x_{j}^{\prime}\right|,
$$

where we typically set $J=5000$. The formulation in (14) further permits the evaluation of the CRPS for discrete distributions such as an ensemble (Grimit et al., 2006). For multivariate distributions, the energy score (ES) provides a similar measure of predictive skill (Gneiting and Raftery, 2007). It is given by

$$
\operatorname{es}(G, \boldsymbol{y})=\mathbb{E}\|\boldsymbol{X}-\boldsymbol{y}\|-\frac{1}{2} \mathbb{E}\left\|\boldsymbol{X}-\boldsymbol{X}^{\prime}\right\|
$$

where $\|\cdot\|$ denotes the Euclidean norm, and may be approximated as the CRPS above.

It has been noted (Pinson and Tastu, 2013) that the sensitivity of the energy score to misrepresentation of the correlation structure is rather limited. As an additional score, we therefore consider the Dawid-Sebastiani (DS) score which depends on the predictive mean vector $\boldsymbol{\mu}_{G}$ and the predictive covariance matrix $\boldsymbol{\Sigma}_{G}$ of the multivariate predictive distribution $G$ via

$$
\operatorname{ds}(G, \boldsymbol{y})=-\log \operatorname{det} \boldsymbol{\Sigma}_{G}-\left(\boldsymbol{y}-\boldsymbol{\mu}_{G}\right)^{t} \boldsymbol{\Sigma}_{G}^{-1}\left(\boldsymbol{y}-\boldsymbol{\mu}_{G}\right)
$$

(Dawid and Sebastiani, 1999; Gneiting and Raftery, 2007). For multivariate Gaussian distributions such as the spatial NGR predictive distributions, the Dawid-Sebastiani score is equal to the logarithmic or ignorance score and may be calculated directly. For spatial BMA, the mean and covariance matrix may be calculated as follows. Let $\mathbf{Y}$ be a random vector which distribution is given by a mixture of $M$ Gaussian distributions each with mean $\boldsymbol{\mu}_{m}$, covariance $\boldsymbol{\Sigma}_{m}$ and weight $\omega_{m}$ for $m=1, \ldots, M$. Then is holds that,

$$
\mathbb{E}\left(Y_{i}\right)=\sum_{m=1}^{M} \omega_{m} \mu_{m i}
$$

and

$$
\mathbb{E}\left(Y_{i} Y_{j}\right)=\sum_{m=1}^{M} \omega_{m}\left(\left(\boldsymbol{\Sigma}_{m}\right)_{i j}+\mu_{m i} \mu_{m j}\right) .
$$

The former formula can now be used to calculate the mean $\boldsymbol{\mu}_{G}$ while the covariance matrix may be calculated by noting that $\left(\boldsymbol{\Sigma}_{G}\right)_{i j}=\mathbb{E}\left(Y_{i} Y_{j}\right)-\mathbb{E}\left(Y_{i}\right) \mathbb{E}\left(Y_{j}\right)$. When $\Sigma_{G}$ must be estimated non-parametrically from a 
sample, such as for ECC, the calculations may be numerically unstable. In this case, we add 0.00001 to all elements on the diagonal in order to improve the numerical stability (Rasmussen and Williams, 2006).

Finally, we provide some error measures of the deterministic forecasts that are obtained as functionals (e.g. mean or median) of the predictive distributions. For univariate probabilistic forecasts, the mean absolute error (MAE) and the root mean squared error (RMSE) assess the average proximity of the observation to the center of the predictive distribution. The absolute error is calculated as the absolute difference between the observation and the median of the predictive distribution while the squared error is calculated using the mean of the predictive distribution (Gneiting, 2011). The Euclidean error (EE) is the natural generalization of the absolute error to higher dimensions. It is given by the Euclidean distance between the observation and the

median of the predictive distribution. The median of a multivariate predictive distribution is estimated using the functionality of the R-package ICSNP. 